\title{
Table ronde : mettre en scène Shakespeare aujourd'hui
}

\section{(2) OpenEdition \\ 1 Journals}

\section{Édition électronique}

URL : http://journals.openedition.org/shakespeare/532

DOI : $10.4000 /$ shakespeare. 532

ISSN : 2271-6424

Éditeur

Société Française Shakespeare

\section{Édition imprimée}

Date de publication : 1 novembre 1989

Pagination : 177-191

\section{Référence électronique}

"Table ronde : mettre en scène Shakespeare aujourd'hui », Actes des congrès de la Société française Shakespeare [En ligne], 6| 1989, mis en ligne le 01 janvier 2007, consulté le 06 mai 2019. URL : http:// journals.openedition.org/shakespeare/532; DOI : 10.4000/shakespeare.532 


\section{SOCIETE FRANCAISE SHAKESPEARE}

\section{Actes du Congrès - 1984}

\section{LIEU ET TEMPS}

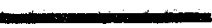

Directeur de la publication Jean FUZIER 


\section{Table ronde : Mettre en scène Shakespeare aujourd'hui}

Participent à cette table ronde :

- Jean-Pierre Vergier, décorateur de Georges Lavaudant, à Grenoble.

- Guy-Claude François, décorateur de presque tous les spectacles de Mnouchkine, et notamment de Richard II, La Nuit des Rois et Henry $I V, 1^{e}$ partie, et auteur du nouveau dispositif d'Avignon.

- Hortense Guillemard, décoratrice du Hamlet mis en scène par ellemême et François Marthouret.

- Georges Lavaudant, metteur en scène.

- Yannis Kokkos, décorateur, entre autres, de Hamlet, mis en scène par A. Vitez à Chaillot il y a deux ans.

- Georges Banú, enseignant à l'Institut Théâtral de Paris III qui collabore régulièrement aux Voies de la Création Théâtrale et qui vient d'écrire un article sur la scénographie contemporaine, dans Theatre Drama Review 
Jean-Michel Déprats.

Q. La première question s'adresse plus à G.-C. François et à Y.-Kokkos car, au Théâtre du Soleil chacun des projets s'accompagne en général d'un remodelage complet du lieu : le cratère de l'Age d'Or, les deux scènes qui se faisaient face dans Mephisto. De même, il y a eu un travail scénographique pour la première saison des spectacles de Chaillot où les trois pièces se sont données dans un même dispositif : une forêt, une scène en éperon. Pour la deuxième saison, tous les spectacles se sont joués dans un nouveau dispositif : la construction d'un théâtre dans le théâtre. Voici donc ma question: tenez-vous à définir votre travail plus en termes de scénographie que de décoration?

G.C.F. Souvent le mot décorateur est pris dans un sens péjoratif, aussi ma fierté m'interdit de l'être. Mais en fait, j'avoue ne pas savoir définir l'un ou l'autre. Je constate des définitions différentes. Comme je suis très peu attaché au statut quel qu'il soit, je peux difficilement répondre à cette question.

Q. $\quad$ Ma question est peut-être mal formulée. Dans mon esprit, le décorateur est l'auteur du décor dans un lieu scénique, le scénographe tel que je l'entends est celui qui conçoit l'aménagement de l'ensemble de l'espace théâtral.

G.C.F. Dans ce sens, je me sens scénographe puisqu'au Théâtre du Soleil, on conçoit la salle dans son intégralité, et que par ailleurs, je construis des théâtres. Mais je n'y attache pas trop d'importance.

Y.K. Moi non plus. C'est souvent la première question qui m'est adressée, alors je n'y réponds plus. Mais le mot scénographie, qui est celui qui domine plus ou moins actuellement, définit peut-être d'une façon plus précise ce que nous faisons: on ne décore pas les spectacles, on fait les images pour la scène, on écrit sur la scène.

G.B. Je voudrais revenir au décor des Shakespeare d'Ariane Mnouchkine et d'Hamlet, il me semble que quelque chose les réunit. Dans les deux cas, on a fait référence au théâtre élisabé- 
thain, et bien que camouflée, on retrouve très nettement une référence à un lieu traditionnel. Dans la scène de Mnouchkine, il y a la scène de kabuki, mais modifiée, renversée, et dans Hamlet il y a aussi un effet situationnel, un effet de perspective très appuyé dans cette scène à l'italienne.

Y.K. Ce que j'ai essayé de faire avec Hamlet c'est d'affronter Shakespeare qui est finalement un compagnon pour nous tous, non pas d'une manière historique - ne pas considérer que l'espace shakespearien existe dans le théâtre élisabéthain, mais que l'espace shakespearien existe dans le texte, c'est-à-dire dans l'écriture. Ce que m'intéressait le plus dans Hamlet, ce n'était pas de recréer un nouveau Shakespeare dans un imaginaire romantique, comme c'était le cas il y a quelques années : l'image romantique du sombre,des tourments d'une âme, de l'histoire dans l'espace nocturne, mais de trouver un espace où règne l'esprit de la Renaissance dans ce qu'il avait d'approche philosophique. Hamlet est finalement la pièce de théâtre qui contient toutes les autres, la tragédie grecque comme les formes de théâtre les plus modernes. C'est une pièce-charnière dans le théâtre européen. J'ai cherché à faire un espace qui raconte tout ce qui peut être possible à l'intérieur, et que les acteurs, la manière dont ils se déplacent, deviennent les dessins vivants d'un univers clair, que puisse se dessiner un espace ouvert mais qui en même temps imprime une image très forte. En fait il ne s'agit pas d'un espace neutre, j'ai horreur des espaces neutres ou des choses qui doivent se voir le moins possible. Ce n'est pas une manière d'aborder le théâtre. Le plus important et le plus difficile à faire c'est quand il n'y a rien.

Le projet global d'il y a deux ans, ce décor unique avec fosse de tombeau pour les trois spectacles colorait l'espace d'une manière différente. C'était un parti-pris partiellement réussi. La plus grande difficulté que l'on a rencontrée était liée à la salle de Chaillot en elle-même : c'était un problème acoustique. Comment faire entendre les textes dans la grande salle telle qu'elle existait avec les structures métalliques. Puisque l'on ne travaillait que du théâtre de texte, cela a été presque une nécessité de faire que les gens voient bien et entendent bien. Pour cela, il n'y a pas un théâtre idéal, mais le théâtre frontal sur 
lequel peut s'ouvrir la totalité de l'espace est une solution. Il y a d'autres réponses, mais c'était celle qui, pour un grand espace, s'imposait le mieux. En retrouvant l'image traditionnelle de Chaillot, les spectateurs pouvaient retrouver l'image habituelle du théâtre. C'est une raison pour laquelle j'ai raccordé l'espace scénique avec le bâtiment lui-même. Comme le grand spectacle de transformation, c'était Hamlet, la pièce sur le théâtre, cela méritait bien de construire un théâtre. Une liaison a permis de transformer cet espace pour le second spectacle en faisant un décor dans ce cadre à l'italienne qui a été détruit il y a quelque temps.

Q. Dans la dramaturgie de Shakespeare, vous ne trouvez pas qu'il y a un lien essentiel avec les conditions physiques de représentation de la scène élisabéthaine?

Y.K. Je dirais que j'y trouve une liaison avec le théâtre, c'est-àdire entre les acteurs et l'espace. Il impose cela d'une manière impérative. Tout est absolument possible. Quelque chose me fascine toujours par exemple dans le Conte d'Hiver. Le début de la pièce présente un personnage important, un noble. On a l'impression qu'on va le suivre pendant la pièce entière. L'action continue, il part avec l'enfant sur un bateau qui fait naufrage. Ils arrivent sur une île, soudain un ours sort et le mange. On ne le voit plus. C'est extraordinaire de pouvoir faire exister quelqu'un si fortement dans l'écriture et de le faire disparaitre si vite. C'est absolument contre les lois de la dramaturgie. Tout est de la même veine dans ce théâtre, donc il faut trouver des espaces dans lesquels ces choses puissent s'épanouir. Généralement dans mon approche de Shakespeare, je crois que je ne ferai jamais de décor illustratif où l'anecdote aurait place. En fait j'essaierai toujours de trouver des espaces qui peuvent respirer avec un texte. C'est ce que j'ai voulu faire avec Hamlet qui était un peu un stade d'aboutissement de ce que j'ai envie de faire avec les pièces de Shakespeare : donner la primauté au mouvement de l'acteur.

Q. Je vais poser la question aux autres participants: quel rapport ont-ils avec la scène élisabéthaine? Dans le dispositif de la Cartoucherie, on retrouve l'espace nu, peu d'éléments 
décoratifs, vous fonctionnez essentiellement sur l'imagination du verbe et par la parole de l'acteur. Est-ce pertinent dans votre réflexion de vous interroger quand vous montez un Shakespeare, sur votre rapport à la scène élisabéthaine? Entre parenthèses, j'aimerais bien savoir ce qu'on dit de la scène élisabéthaine dans les différentes écoles d'art plastique, car beaucoup de points sont controversés : l'existence de cette fameuse scène intérieure, de l'espace de découverte...

G.C.F. C'est vrai que lorsqu'on est à l'école, on apprend tout ça : le théâtre à l'italienne, le théâtre élisabéthain... Et au fur et à mesure de l'expérience concrète du plateau et de la dramaturgie, je me rends compte qu'il n'y a absolument aucun rapport entre la dramaturgie et l'architecture théâtrale. Ce n'est pas seulement valable pour la scène élisabéthaine, mais pour l'ensemble des scènes. Si vous vous amusez à superposer, par exemple, tous les théâtres occidentaux qui ont été réalisés depuis que le théâtre existe, vous vous rendez compte que toutes les thèses émises dans les écoles, dans les livres... ne portent que sur des choses qui ne sont finalement que des détails pour les gens de théâtre - des détails parfois importants, parfois contraignants, mais des détails du genre : rideaux, loges, fosses d'orchestre... La seule chose qui est constante et valable est la géométrie qu'un acteur impose face à une assemblée qui l'écoute et qui le regarde - géométrie, c'est à dire tout ce que cela signifie pour un architecte : la trame, la position des gens... Je suis de plus en plus convaincu qu'il n'y a pas de rapport entre la dramaturgie et le théâtre, et que l'architecture théâtrale n’a jamais été influencée, inspirée que par la définition sociale de l'époque. Ceci est valable non seulement pour le théâtre, mais pour tout monument, tout édifice public. J'en veux pour preuve que lorsque on veut monter Tchékhov, Shakespeare ou Molière, on ne se pose jamais le problème. Il y a d'autres sources d'inspiration, mais pas celle de l'architecture.

Q. On a tendance à penser que le mode de rapport entre le public et l'acteur se trouvant sur une scène qui s'enfonce vers le public, n'est pas le même que celui que l'on a avec une scène frontale. 
G.C.F. Si dans le théâtre grec on a le chœur avec la position du coryphée, dans le kabuki il y a une passerelle. Vous retrouvez d'une façon différente ce souci de proximité. Mais parfois, on veut une distance.

Quant à ce qu'on appelle la scène intérieure, c'est le mot que l'on utilise pour la scène élisabéthaine, un scénographe n'a qu'un seul souci : créer un espace qui puisse être à la fois celui qui contient l'âme dans ce qu'elle a de plus intime, et qui puisse s'ouvrir sur des infinis galactiques. Ce n'est qu'une façon mécanique de répondre à ce problème - qui est un problème constant - que l'on voit derrière la phrase de chaque grand auteur. Quand les auteurs disent: "La scène se passe dans un salon", "la scène se passe dans un palais ", si vous vous amusez à dessiner cela, souvent vous vous retrouvez devant un objet dont la quotidienneté bannit toute la dimension théâtrale de l'œuvre. Et en fait, il ne fait qu'indiquer à lui-même et au maître d'œuvre une limite - non pas une limite d'aboutissement, ni de départ, mais quelque chose qui ouvre à l'imagination.

Y.K. Quand je travaille sur Shakespeare, je ne pars pas de ce qu'était l'espace élisabéthain, en essayant de transposer cet espace pour jouer, je pars directement de l'œuvre elle-même. Les solutions que je vais trouver n'existent pas uniquement dans ce qui est information de l'écriture, mais dans ce qui est poésie : de quelle manière faire s'épanouir la poésie, comment ne pas la bloquer.

H.G. Il y a un autre plan qui est peut-être moins conceptuel. Quand on s'attaque à une pièce, quelle qu'elle soit, on tient compte de l'entreprise en elle-même et de ce type d'entreprise découlent des possibilités différentes. Je ne travaille ni à Chaillot ni avec Mnouchkine mais de façon plus modeste. Il y a des données concrètes, réalistes, mais très importantes d'où découlent les possibilités d'inventer et d'imaginer. Je ne me suis absolument pas posé la question de l'élisabéthain ou du non élisabéthain. Je démarrais un spectacle sur une grande scène et j'ai été obligée de m'adapter aux Bouffes du Nord, ce qui n'avait rien à voir : d'une ouverture de 14 mètres de cadre de scène à un théâtre en promiscuité totale puisqu'au Bouffes il n'y a pas 
de plateau et qu'on joue au milieu du public. Un autre élément dont j'avais à tenir compte était le budget. On ne peut pas parler d'une totale liberté. On l'a au niveau de l'imaginaire, mais je pars de données concrètes. Et comme dit Yannis, après ces données concrètes, il y a la pièce qui déclanche des idées.

(quelques diapositives sur les spectacles)

Q. Quelques mots sur le dernier décor dont il a été question, celui d'Hamlet mis en scène par Hortense Guillemard et François Marthouret, dont nous n'avons pas de diapositives. Je vais lire le texte de présentation rédigé par Hortense Guillemard, elle le commentera ensuite :

"Ce lieu unique où va se raconter notre Hamlet je le rêvais navire, îlot de rochers, espace fantasmatique et ruines et mort et aussi vie, puissance arrêtée, pétrifiée. Je le voulais symbolique et surtout pas réaliste. De ce chaos de désirs ne pouvait nấtre qu'un chaos. Murs éboulés ou armée en marche, monument aux morts baroque, ou tas d'êtres sans visages, ils sont une soixantaine, hommes ou femmes aux corps pétrifiés, pris dans la lave peut-être; par groupes ou isolés, murs de corps qui s'avancent vers nous spectateurs comme des voyeurs aveugles, des témoins muets, des spectres du spectre. Immobiles et inéluctables, ils regardent sans la voir la tragédie. Ils sont mémoire, mais on ne sait trop de quoi, peut-être de tous les Hamlet qui ont précédé celui-là.»

\section{(photos à consulter)}

H.G. J'étais obsédée par le voyeurisme dans Hamlet, par l'espionnage continuel. C'est vrai que je n'ai pas voulu faire une chose abstraite et simplifiée, et que je ne voulais pas non plus quelque chose de réaliste. Une nuit je me suis dit: une montagne de gens va regarder le public en face de lui, et entre les deux va se jouer Hamlet. Les gens sont devenus des cadavres, mais c'était des sortes de mannequins pétrifiés grandeur nature, il y en avait plus de soixante, qui formaient comme une énorme falaise ; une sorte d'architecture, un peu comme Pompéi, de sculpture pétrifiée. A l'intérieur, c'était à la fois le château, l'intérieur 
du château, l'extérieur du château. Et les personnages, Polonius entre autres, espionnaient les autres à l'intérieur de ces autres personnages, c'était un grouillement terrible.

Q. $\quad$ On peut maintenant tenter de répondre à la question centrale qui est de définir le particularisme du travail du plasticien, du scénographe, du décorateur, peu importe, sur une pièce de Shakespeare. On a déjà dit un certain nombre de choses sur la rapidité de l'action, la multiplicité des lieux, la mobilité inscrite dans la dramaturgie et dans le flot des scènes. J'aimerais que chacun tente de répondre à ce point-là : qu'est-ce qui lui apparaît comme spécifique, comme particulièrement marquant dans le travail, ou les travaux, qu'il a faits sur Shakespeare. Puisqu'en dehors des mises en scène que nous avons citées, Yannis Kokkos par exemple a monté bien d'autres Shakespeare que celui dont nous parlons et que récemment, Vitez a monté un opéra, Macbeth de Verdi, dans ses décors.

G.B. Y a-t-il des difficultés inhérentes à Shakespeare pour le scénographe?

H.G. Il y a une difficulté évidente : il y a une évocation de nombreux lieux, et il y a sans arrêt des situations scéniques proposées par le scénario de la pièce qui sont d'une grande difficulté. On passe d'un champ de bataille à une anti-chambre. Si on commence à se poser le problème de cette illustration, c'est totalement impossible. Je ressens la même choses que Yannis qui disait qu'il n'avait nullement envie de proposer une illustration anecdotique.

Y.K. C'est faisable, mais est-ce intéressant?

H.G. Non je ne crois pas.

Q. $\quad$ Il faut rappeler que l'histoire du décor au XIX $\mathrm{X}^{\mathrm{e}}$ par rapport à Shakespeare est un immense malentendu. Pour pouvoir changer de décor, il fallait couper la moitié ou les trois-quarts du texte.

G.C.F. Il me semble évident que Shakespeare impose un décor unique, ce qui n'est peut-être pas particulier à Shakespeare, d'ailleurs. 
Y.K. Je dirai qu'il y a presque un combat à mener pour maintenir absolument la spécificité du théâtre, son originalité d'art. Et je pense que Shakespeare est notre modèle.

G.B. Ce qui m'a frappé dans ces différentes versions, c'est que, par rapport à l'ancien type de mise en scène de Shakespeare, il y avait d'un côté le mouvement, mais d'un autre côté le scénario se manifestait par un dispositif fixe très puissant qui investissait les regards tout au long de la représentation, aussi bien dans Hamlet aux Bouffes, qu'à Chaillot, que dans Macbeth. Il y avait la volonté, non pas de donner un espace qui guide seulement le mouvement, mais qui permette au regard des spectateurs de s'accrocher, d'investir une image fixe.

Q. $\quad$ Ce que René Allio appelle le paysage mental. La transformabilité se fait à l'intérieur d'un élément fixe et non pas par changement de décor.

G.C.F. D'une certaine manière, c'est plus facile avec Shakespeare, car on ne doit tenir compte que de sa poésie. On ne peut pas faire autrement, alors que parfois des auteurs fournissent des indications qui sont très précises. C'est à l'intérieur du texte de Shakespeare que l'on trouve l'essentiel. Je me rappelle une ou deux phrases : "le soleil de Bolingbroke" dans Richard II m'a tout de suite donné une idée, dans Henry $I V$, dès le début de l'acte IV ou V, avant la bataille: "quel oil sanglant me regarde ». C'est une indication de décor qui est bien plus évidente que : "la scène se passe dans le salon de la marquise ".

Q. C'est l'idée connue que le décor est dans le texte, et donc que le scénographe n'a pas à rivaliser avec la poésie du texte. Cela veut-il dire que l'on tend vers des espaces plus dénudés en faisant référence au livre du Brook-que pour d'autres auteurs?

G.C.F. C'est ce que disait Yannis tout à l'heure. On peut dire qu'un espace vide est un espace neutre, auquel cas, c'est souvent horrible. Mais il est vrai que le vide est ce qu'il y a de plus difficile à faire. 
Q. Un problème est en filigrane depuis longtemps : c'est le rapport à la mise en scène, plutôt que le rapport au metteur en scène. Hortense Guillemard, est-ce facile d'avoir les deux rôles dans un même spectacle?

H.G. Ce n'est pas viable. C'est un peu une folie. On ressort bloqué et frustré des deux côtés parce qu'on a besoin d'autonomie et de liberté dans chacun de ces postes et que la juxtaposition est une difficulté épouvantable. La main gauche brûle toujours la main droite. Je sais que je ne referai jamais ça! Cela dit, c'est passionnant à vivre quand même, même si au bout du compte, j'ai l'impression d'une erreur, car c'est trop à partì d'un certain stade du travail. C'était merveilleux dans toute la conception et justement là, c'était un apport formidable que d'être décoratrice. Mais il faut préciser que je n'étais pas toute seule à la mise en scène, nous étions deux, donc, il y avait tout de même un autre regard, une autre personne, une autre distance. Ce n'était pas l'horreur absolue d'être toute seule des deux côtés. Mais en cours de concrétisation des idées, des fantasmes, j'ai trouvé cela de plus en plus dur. J'ai eu du mal à transmettre, en tant que metteur en scène, le projet de mise en scène qui était, je pense, inclu dans le décor. J'ai eu du mal à résister en tant que décoratrice.

Q. «Le projet de mise en scène était inclu dans le décor ». Cela vous parait-il toujours vrai ? Est-ce souhaitable?

Y.K. Ce qui se passe avec un metteur en scène est une sorte d'alchimie, de désirs parallèles. A un certain moment, il y a beaucoup de tentations, certaines que l'on trouve justifiées, d'autres moins. Quelquefois, on arrive à ne plus se parler du tout, et on fait des choses parallèlement dans l'espace que l'on propose. Et il fait sa mise en scène à partir des données déjà existantes. D'autres fois, c'est lui qui sent beaucoup plus une direction du spectacle. Je crois que finalement, le théâtre est un travail extrêmement solitaire qui se fait à plusieurs. Plus on se maintient dans la solitude artistique, plus on la partage, plus on va loin. Si je peux utiliser une image qui semble exprimer le mieux ce que nous faisons : c'est un peu comme le jazz. Il y a des solis- 
tes qui travaillent le plus loin possible dans leur propre discipline, et à la fin tout le monde joue la même musique. Ce qui compte est ce qu'on entend. Tout le monde s'efface devant cette image de spectacle qui rassemble, mais qui inclut la totalité des univers particuliers. Cela ne veut pas dire effacement. Ces états particuliers se conjuguent et se fondent avec le travail de la mise en scène qui s'occupe plus particulièrement de l'acteur et de la transmission de l'idée du spectacle par les acteurs.

Q. Si on se référe à la différence de pratique selon les troupes, dans certains cas, le décor est inventé avant le début des répétitions, donc propose, impose sa structuration de l'espace aussi bien que sa circulation.

G.C.F. C'est ce qui se passe au Théâtre du Soleil. Les répétitions commencent en même temps que la construction du décor. Mais je distinguerai deux missions dans le travail des scénographes. Une qui se fait avec le metteur en scène, qui est une sorte de passerelle entre la poésie et l'image, où le metteur en scène a une importance capitale dans la mesure où c'est lui qui a choisi, il faut le rappeler, la pièce, son signe, et sa façon de l'exprimer. La deuxième mission qui est très claire et très solitaire, est de faire exprimer, de maîtriser la matière, les couleurs, les formes, les volumes, pour en faire un espace. La plupart des décorateurs forment avec les metteurs en scène une espèce de couple, et comme tous les mariages réussis, l'osmose des regards réciproques et simultanés fait qu'il y a réellement une difficulté à dissocier les deux.

G.B. Y a-t-il des demandes du metteur en scène qui seraient faites pour votre travail, ou y a-t-il des propositions de vous vers l'autre?

J.P.V. C'est très variable. Parfois Georges a des idées de décor, et parfois, c'est moi qui les ai. C'est difficile de changer de metteur en scène. J'ai essayé... et je suis revenu.

G.L. J'ai trouvé très intéressant ce qu'a dit Yannis sur l'addition des solitudes. C'est là qu'est la solution. Au bout d'un moment on ne se surprend plus. Y a-t-il une spécificité shakespearienne 
du décor? Je ne le crois pas du tout, car on se choisit un décorateur, on sait quel est son travail plastique. Si je suis allé vers Vergier, ce n'était pas un hasard, et si je suis resté avec lui, c'est que je voyais bien que son travail plastique me motivait, me stimulait. Mais ensuite, on se trouve devant le problème inverse : comment continuer à se surprendre, à approfondir mutuellement nos arts? Sinon, ce sont nos solutions plastiques, nos univers que l'on répète. Chaque fois qu'un nouveau projet démarre, comment refaire des appels de désir, ouvrir des portes? Comment avoir l'impression que l'on ne va pas refaire la même chose? Je parle de Jean-Pierre, mais c'est vrai d'un acteur, particulièrement d'Ariel. Il a fait la moitié de la mise en scène de Richard III, et Jean-Pierre a fait plus: il a fait les costumes, le décor, les éclairages. Avec Jean-Pierre, la question est : comment peut-on se surprendre. ? Je sais ce qu'il aime : on a toujours fait des décors uniques dès le début, ce n'est pas particulier à Shakespeare. Il n'aime pas qu'il y ait beaucoup d'objets sur le plateau. Mais chaque fois, je voudrais changer. C'est formidable d'avoir une équipe dans le théâtre, d'avoir un collectif d'acteurs, de décorateurs..., mais il faut voir quand on commence à se répéter, quand on est le prisonnier de nos propres stylistiques. Je n'ai pas envie de travailler avec un autre décorateur, mais j'aimerais savoir comment on peut casser des habitudes.

G.B. Sachant que Georges Lavaudant n'aime pas le changement, que vous ne voulez pas casser ses habitudes, quelles sont vos constatations sur son travail de metteur en scène? Quelles sont ses demandes?

J.P.V. Je réponds déjà sur le décor: si on n'avait pas joué à Avignon, on aurait fait un autre décor pour Richard III. On est un peu lié aux salles dans lesquelles on travaille. A Grenoble, on joue dans une très grande salle qui a une des plus belles cages de scène, bien qu'elle manque de profondeur. Par contre, on a un très mauvais rapport scène-salle, ce qui arrive souvent dans les théâtres contemporains. On travaille notre scénographie en fonction de cette salle. Mais comme on tourne pas mal, on a des problèmes de montage. Je fonctionne beaucoup sur des 
choses que je m'interdis, des allergies : de ce que j'appellerai le décorum, ou des choses très décoratives. On a parfois des tensions sur les objets, Georges a tendance à amener des objets sur scène que j'ai envie de retirer.

G.L. Dans le théâtre en France et en Europe, il y a un éventail de plus en plus large d'esthétiques. Par exemple, Jean-Pierre déteste le blanc, les décors clairs. Je trouve formidable que Yannis travaille là-dessus.

Y.K. Il y a une série de dogmes, parfois universitaires, sur ce qu'est l'esthétique au théâtre. Et à un certain moment, il faut absolument s'y plier, sous peine de mort. Je travaille dans le théâtre en France depuis vingt ans environ, et j'ai connu des blocages extraordinaires et des sanctions terribles à partir du moment où certaines personnes essayaient de faire ce qui n'était pas accepté ou admis. Et ces spectacles qu'on accuse d'être à la mode, qu'est-ce que cela veut dire ? Sentir un courant, essayer de le matérialiser sur scène. C'est formidable qu'un spectacle puisse capter ce qui est à la pointe à un certain moment, et le rende. Je trouve qu'aujourd'hui, on souffre moins de ce regard dogmatique sur le plan de l'esthétique.

G.L. Dans l'espace scénique que Jean-Pierre propose, je lui dis tout ce qui ne me plaît pas, mais au bout d'un moment, je lui dis : " prends tes responsabilités, en dernier ressort, c'est ton travail ». C'est la règle du jeu qu'on s'est toujours fixée....

Il y a aussi des contraintes pour les costumes, surtout shakespeariens. Par exemple, le costume de la reine Marguerite doit bien peser $20 \mathrm{~kg}$, avec corset... La première fois que Philippe Morier-Genoud l'a passé, il voulait tuer Vergier! Mon devoir était de faire travailler cette chose-là. Je ne voulais pas a priori dire non. Je voulais essayer. Et s'il y a un énorme problème, on revient à Jean-Pierre.

G.B. Je voudrais parler de Macbeth, l'opéra de Verdi, écrit à partir d'une ouvre de Shakespeare. Que prend-on en compte? Part-on du livret, de la musique ? En voyant le décor de Yannis Kokkos, j'ai retrouvé un peu la perspective du décor d'Hamlet. 
On retrouve ce besoin d'éloignement. Dans les deux décors, il y a une violente ligne de fuite. L'angle d'inclinaison était très fort.

Y.K. Le problème est un peu plus vaste. Fait-on le même décor pour l'opéra et pour le théâtre ? J'ai fait deux opéras d'inspiration shakespearienne : Lear à l'Opéra de Paris, et Macbeth. Plus je fais d'opéras, plus je pense que ce n'est pas du tout.le même type de décor, pas sur le plan pratique, mais sur le plan esthétique général. Par exemple, je ne ferais jamais le décor que j'ai fait pour Macbeth à l'Opéra sur une scène de théâtre. Ma conception d'Hamlet était assez proche de celle des chutes de corps. Pour Macbeth, j'ai dû prendre en compte la dimension romantique de Verdi par rapport à Shakespeare, et l'intelligence tout à fait remarquable de son interprétation. Contrairement à ce que je pensais, la parole remplit l'espace de la scène alors que la musique a besoin de s'accrocher à quelque chose de plus réel, comme le cinéma. Si dans le cinéma on ne fait pas du tout de réalisme ou si on part dans des choses où le naturel n'existe pas, on a besoin de s'accrocher à du vraisemblable, à de la matière.

G.L. Jean-Pierre a eu trois expériences: Iphigénie avec Bruno Bayen et nos deux expériences - Roméo et Juliette de Gounod et un Mozart. Je partagerais un peu ton point de vue sauf en ce qui concerne Mozart. Pour Mozart, il y a encore moyen de faire du théâtre. Et maintenant, je me rends compte que je n'aimerais pas faire d'opéra, sauf Mozart.

Y.K. L'espace de la mise en scène pure est plus réduit à l'opéra.

G.L. Ce n'est pas le même travail.

Q. $\quad$ Puisque vous vous êtes mis à faire des décors pour l'opéra, n'entrez-vous pas dans un système qui a déjà son inertie, totalement différente de celle du théâtre?

Y.K. Absolument. Les économies de théâtre sont multiples. J'adore toutes les formes de théâtre, même les plus ringardes. Ce qui est intéressant, c'est exalter un genre : de quelle manière le genre dans lequel on travaille peut briller, à travers sa convention, 
mais en même temps à travers sa modernité. Quelles sont les bonnes mises en scène pour les gens qui aiment la musique et l'opéra? Ils aiment être émoustillés par ce qu'ils estiment être des innovations. Par exemple Rigoletto dans les bas-fonds de Chicago. Cela ne m'intéresse pas parce que c'est une esthétique plaquée. Ce qui m'intéresserait, ce serait de faire Rigoletto dans je ne sais quel style inventé et qu'on puisse penser à Chicago à travers cela. Comment donner une image de la modernité en regardant le passé. C'est pourquoi nous revenons à Shakespeare. Ses pièces correspondent à un regard intense sur le passé qu'on peut regarder avec le regard d'aujourd'hui. 